\title{
Characterization of Pseudomonas aeruginosa isolated from chronically infected children with cystic fibrosis in India Gunjan Agarwal ${ }^{1}$, Arti Kapil*1, Susheel Kumar Kabra ${ }^{2}$, Bimal Kumar Das ${ }^{1}$ and Sada Nand Dwivedi ${ }^{3}$
}

Address: ${ }^{1}$ Department of Microbiology, All India Institute of Medical Sciences, Ansari Nagar, New Delhi-110029, INDIA, ${ }^{2}$ Department of Pediatrics, All India Institute of Medical Sciences, Ansari Nagar, New Delhi-110029, INDIA and ${ }^{3}$ Department of Biostatistics, All India Institute of Medical Sciences, Ansari Nagar, New Delhi-110029, INDIA

Email: Gunjan Agarwal - gunjan777@yahoo.com; Arti Kapil* - akapil_micro@yahoo.com; Susheel Kumar Kabra - skkabra@hotmail.com; Bimal Kumar Das - tezpur@yahoo.com; Sada Nand Dwivedi - dwivedi7@hotmail.com

* Corresponding author

Published: 21 July 2005

BMC Microbiology 2005, 5:43 doi:10.1 |86/|47|-2/80-5-43
Received: 03 April 2005

Accepted: 2I July 2005

This article is available from: http://www.biomedcentral.com/I47I-2/80/5/43

(c) 2005 Agarwal et al; licensee BioMed Central Ltd.

This is an Open Access article distributed under the terms of the Creative Commons Attribution License (http://creativecommons.org/licenses/by/2.0), which permits unrestricted use, distribution, and reproduction in any medium, provided the original work is properly cited.

\begin{abstract}
Background: Pseudomonas aeruginosa is the leading cause of morbidity and mortality in patients with cystic fibrosis (CF). With chronicity of infection, the organism resides as a biofilm, shows multi-drug resistance, diversifies its colony morphology and becomes auxotrophic. The patients have been found to be colonized with multiple genotypes. The present work was carried out to characterize $P$. aeruginosa isolated from children with cystic fibrosis using phenotypic and genotypic methods.
\end{abstract}

Results: We studied 56 patients with CF attending the Pediatric Chest clinic at All India Institute of Medical Sciences, New Delhi, India during August 1998-August 200I. These patients were regularly followed up at the clinic. Out of 56 patients, 27 were culture positive for $P$. aeruginosa where 8 were chronically infected (Groupl) and 19 were intermittently colonized with the organism (Group2). Patients under Groupl had significantly higher rates of hospitalization, death and colonization with different colony morphotypes $(p<0.05)$. The isolates from Groupl patients were the positive producers of extended spectrum beta lactamase. A total of 5 auxotrophs were recovered from 2 patients where one was chronically infected with $P$. aeruginosa and the other was a recently enrolled patient. The auxotrophs had the specific requirement for methionine and arginine. Molecular typing revealed 33 ERIC-PCR (EI-E33) and 5 PCR-ribotyping (PI-P5) patterns. By ERIC-PCR, 4 patients were colonized with 2-4 genotypes and the remaining 23 patients were colonized with the single genotype.

Conclusion: With chronicity of infection, $P$. aeruginosa becomes multidrug resistant, diversifies its colony morphology, acquires mucoidity and shows auxotrophy for amino acids. The chronically infected patients can be colonized with multiple genotypes. Thus in a particular clinical set up, high index of suspicion should be there for diagnosis of CF patients so as to prevent the delay in diagnosis and management of CF patients. 


\section{Background}

Cystic fibrosis (CF) is the recessive genetic disorder of Caucasian race. CF in Asians and Indians was thought to be almost nonexistent in the past. However, recent reports have suggested CF to be more common in Indian population than previously thought. For proper management, it is important to diagnose the infection in the patients with CF [1-3].

The mutations in the cystic fibrosis transmembrane regulator gene in CF patients lead to recurrent and chronic respiratory tract infections, which serves as a major cause of morbidity and mortality [4]. If appropriate antibiotics are used, the quality of life improves in CF patients. Thus, the knowledge of the etiological agents and their antimicrobial susceptibility can help in planning the appropriate antibiotic therapy to be instituted in CF patients.

Infection due to Pseudomonas aeruginosa ( $P$. aeruginosa) has been implicated as a major cause of morbidity and mortality in patients with CF [5-8]. Once P. aeruginosa colonizes the respiratory passages, it is rarely possible to eradicate it. Prolonged and repeated administration of drugs lead to the development of multidrug resistance in $P$. aeruginosa strains. To detect emergence of the resistant strains, it is important to monitor the antibiotic susceptibility pattern of $P$. aeruginosa isolates. Increased resistance to gentamicin, quinolone and cephalosporins has been reported in chronically infected patients $[9,10]$.

With severity of pulmonary infection, the organism undergoes phenotypic modification, which helps in its persistence under adverse environment of the respiratory tract. The organism diversifies into different colony morphotypes [11,12] and develops antibiotic resistance for its survival. This may be due to an adaptation of the organism to the host defense mechanisms and antibiotic selective pressure [13]. Under recurrent and chronic endobronchial $P$. aeruginosa infections, the initial colonization is with nonmucoid forms of an organism but with progressive lung tissue damage, it gets converted to the mucoid form. The mucoidity helps an organism to grow as biofilm, which serves as a protective niche and helps to evade the host immune response and action of antibiotics. The organism also becomes auxotrophic for amino acid due to high amino acid concentration in the respiratory passages. The most commonly required amino acid reported in literature is methionine while other amino acids-leucine, arginine and ornithine have been required by a minority of $P$. aeruginosa strains [14].

During the course of infection, the organism may undergo genotypic changes. The chronically infected patients have been found to have long-term colonization by one pre- dominant genotype but variation in genotypes within a single patient has also been observed $[13,15]$.

Since no data is available on the characterization of $P$. aeruginosa from CF patients in India, the present study was undertaken to study phenotypic and genotypic variations of $P$. aeruginosa isolated from CF patients attending the Pediatric Chest Clinic at All India Institute Of Medical Sciences (AIIMS), New Delhi, INDIA.

\section{Results \\ Subjects}

A total of 56 patients with CF regularly attended the Pediatric Chest Clinic between August 1998-August 2001. These patients were followed monthly at the chest clinic. At the time of enrollment, all the patients were below 15 yrs of age (mean age (yrs) \pm standard deviation was 5.33 \pm 4.3 and median age was 4.5$)$. The male: female ratio was 2:1.

Out of 56 patients, culture positive infection was observed in $33(58.9 \%)$ patients of whom $27(81.8 \%)$ were culture positive for $P$. aeruginosa and $6(18.2 \%)$ patients were culture positive for organism other than $P$. aeruginosa where one patient each was colonized with Citrobacter freundii, Staphylococcus aureus, Escherchia coli, Enterobacter sp and two patients were colonized with Klebsiella pneumoniae. The rate of infection with $P$. aeruginosa was significantly higher than the rate of infection with organism other than $P$. aeruginosa in CF patients $(\mathrm{p}<0.05)$. Out of 27 culture positive patients, 8 had chronic infection with the organism and were included in Group1. The remaining 19 patients were intermittently colonized with the organism and were included in Group2.

Table I: Antibiograms

\begin{tabular}{ccc}
\hline Antibiogram & $\begin{array}{c}\text { Susceptibility to } \\
\text { antibiotics }\end{array}$ & Total isolates (\%) \\
\hline A1 & S to all & $102(29.1)$ \\
A2 & R to Genta only & $81(23.1)$ \\
A3 & R to Genta+Cipro & $85(24.3)$ \\
A4 & R to Cipro only & $24(6.9)$ \\
A5 & R to Genta+Pipra & $23(6.6)$ \\
A6 & R to & $24(6.9)$ \\
A7 & Genta+Cipro+CAZ & $5(1.4)$ \\
A8 & R to Genta+CAZ & $6(1.7)$ \\
& R to & \\
\hline
\end{tabular}

Genta: Gentamicin; Cipro: Ciprofloxacin; Pipra: Piperacillin; CAZ: Ceftazidime CAZ:

S: Sensitive; R: Resistant

+ : Indicates "and" 

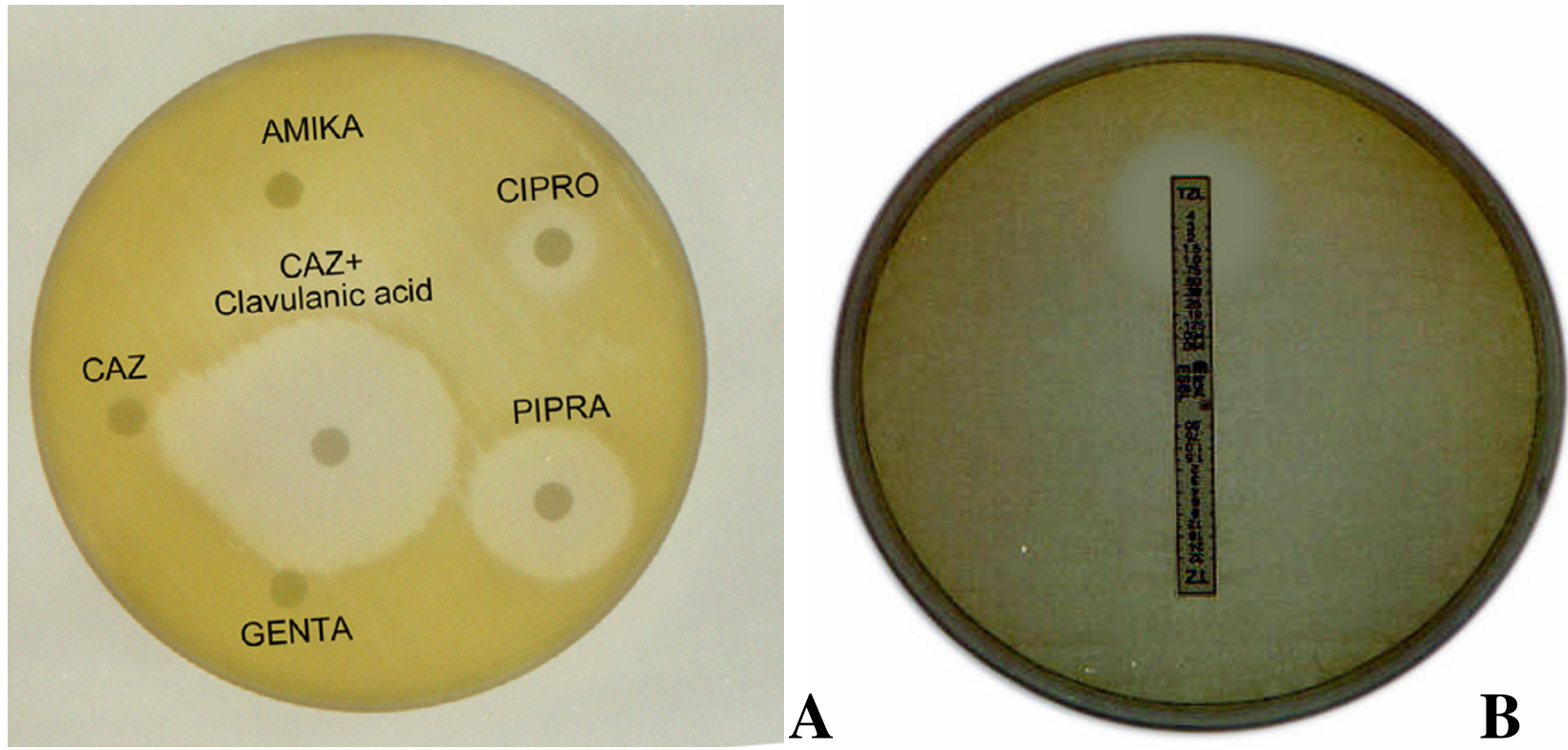

\section{Figure I}

Detection of Extended spectrum $\beta$ lactamase in $P$. aeruginosa isolate from a patient (180/95) by:A. Disc Diffusion method (Amika: Amikacin; Cipro: Ciprofloxacin; Pipra: Piperacillin; Genta: Gentamicin; CAZ: Ceftazidime). B. Etest method (AB Biodisk, Sweden).

During the study period, 9 patients required hospitalization and 4 have died. Of the 4 deaths, 3 occurred during the hospitalization. All the patients who required hospitalization were culture positive for $P$. aeruginosa and were followed daily. The mean duration of hospitalization was 12 days (range was 7-20 days). Out of 9 patients, 6 were included in Group1 and 3 in Group 2. All 4 patients who died, had pulmonary exacerbations and were chronically infected with $P$. aeruginosa. The mortality related to $P$. aeruginosa infection in the study was $14.8 \%$. The frequency of hospitalization and death in chronically infected patients was significantly higher as compared to intermittently colonized patients with P. aeruginosa $(\mathrm{p}<0.05)$.

\section{Antimicrobial susceptibility}

Three hundred and fifty $P$. aeruginosa isolates were recovered from 27 patients where $94(26.9 \%)$ showed the mucoid morphotype (Type5) and the rest 256 (73.1\%) isolates showed nonmucoid morphotype(Type 1-4 and Type 6). The antimicrobial susceptibility was determined by agar dilution method based on NCCLS guidelines and antibiograms were further constructed (Table 1). One hundred and forty three (41\%) isolates were multidrug resistant and showed $\mathrm{A} 3$ and A5-A8 antibiogram. All the isolates were recovered from chronically infected patients.
We further characterized all $29 P$. aeruginosa isolates that showed resistance to ceftazidime and were the positive producers of extended spectrum beta lactamase (ESBL). These isolates were detected as ESBL positive by disk diffusion according to NCCLS guidelines (Fig 1A) and further confirmation was done by Etest method (Fig. 1B). Twenty-four of the $29(82.8 \%)$ ESBL positive isolates were multidrug resistant (A6 antibiogram). All 29 isolates were found sensitive to imipenam and meropenam.

The ESBL positive isolates were recovered from 3 patients (PCC.No. 180/95, 160/98 and 499/00) where 2 (PCC.No. $180 / 95$ and 160/98) were chronically infected and one patient (PCC.No.499/00) was intermittently colonized. Out of 29 ESBL positive isolates, 27 were recovered from the patient (PCC.No. 180/95) during his different followups past two years and 1 isolate each was recovered from 2 patients (PCC.No. 160/98 and 499/00).

\section{Phenotypic characterization of $\mathrm{P}$. aeruginosa isolates Colony morphotypes}

Out of 27 patients infected with $P$. aeruginosa, 7 harbored different colony morphotypes in a single sputum sample while the rest 20 patients harbored Type 1 colony morphotype. All the 7 patients were chronically infected with the organism(Group 1). The rate of colonization with dif- 
Table 2: Distribution of eight antibiograms (AI-A8) among six colony morphological types of $P$. aeruginosa.

\begin{tabular}{|c|c|c|c|c|c|c|c|c|}
\hline \multirow{2}{*}{$\begin{array}{l}\text { Colony } \\
\text { Morphotype } \\
\text { (N) }\end{array}$} & \multicolumn{8}{|c|}{ Total Number of isolate (\%) showing respective Antibiograms } \\
\hline & Al & A2 & A3 & A4 & A5 & A6 & A7 & A8 \\
\hline Type I (194) & $102(52.5)$ & $23(11.8)$ & $27(10.3)$ & $4(1.5)$ & $15(7.7)$ & $14(7.2)$ & $3(1.5)$ & $6(3)$ \\
\hline Type2 (26) & $5(19.2)$ & 0 & $10(38.5)$ & $2(7.7)$ & $\mathrm{I}(3.8)$ & $7(27)$ & $\mathrm{I}(3.8)$ & 0 \\
\hline Type3 (23) & $9(39.1)$ & 0 & $6(26.1)$ & $5(21.7)$ & 0 & $3(13.0)$ & 0 & 0 \\
\hline Type4 (5) & $2(40)$ & 0 & $2(40)$ & $I(20)$ & 0 & 0 & 0 & 0 \\
\hline Type5 (94) & 0 & $39(41.5)$ & 48 (5।.I) & 0 & $7(7.4)$ & 0 & 0 & 0 \\
\hline Type6 (8) & 0 & $5(62.5)$ & I (I2.5) & 0 & 0 & $\mathrm{I}(12.5)$ & 0 & $\mathrm{I}(12.5)$ \\
\hline
\end{tabular}

$N=$ Total Number of isolates

ferent colony morphotypes of $P$. aeruginosa was significantly higher in Group 1 patients $(\mathrm{p}<0.05)$.

Six $(85.7 \%)$ of 7 patients harbored mucoid P. aeruginosa in their respiratory samples and all were chronically infected. The rate of colonization with mucoid P. aeruginosa was significantly higher $(75 \%)$ in chronically infected patients $(\mathrm{p}<0.05)$. Of the 6 patients who were positive for mucoid $P$. aeruginosa, 3 have died.

Out of 350 P. aeruginosa isolates, 194(55.4\%) showed Type 1 morphotype. The distribution of antibiogram among colony morphotypes is shown in Table 2.

\section{Auxotrophy}

Out of $350 P$. aeruginosa isolates, 5 were auxotrophs, which did not grow on minimal agar medium (MAM) and required Mueller Hinton agar (MHA) for their growth. Of the 5 auxotrophs, four (isolate No.2-5) were isolated from the chronically infected patient (PCC.No.180/95) who was diagnosed as CF six years ago. One auxotroph (isolate No.1) was isolated from a recently enrolled patient (PCC.No.499/00). The respiratory samples collected during their subsequent follow-ups were found negative for the presence of auxotrophs. These auxotrophs were recovered when these patients presented with pulmonary exacerbation.

The detection of specific amino acid requirements of auxotrophs revealed that 3(isolate No.1-3) had the specific requirement for arginine (Fig. 2) and 2(isolate No. 4,5) for methionine. The reproducible results were obtained on repeating the experiment thrice.

The resistance against different antibiotics was compared between auxotrophs and prototrophs. Although auxotrophs were small in number, they showed significantly higher resistance against ceftazidime $(60 \%)$ as compared

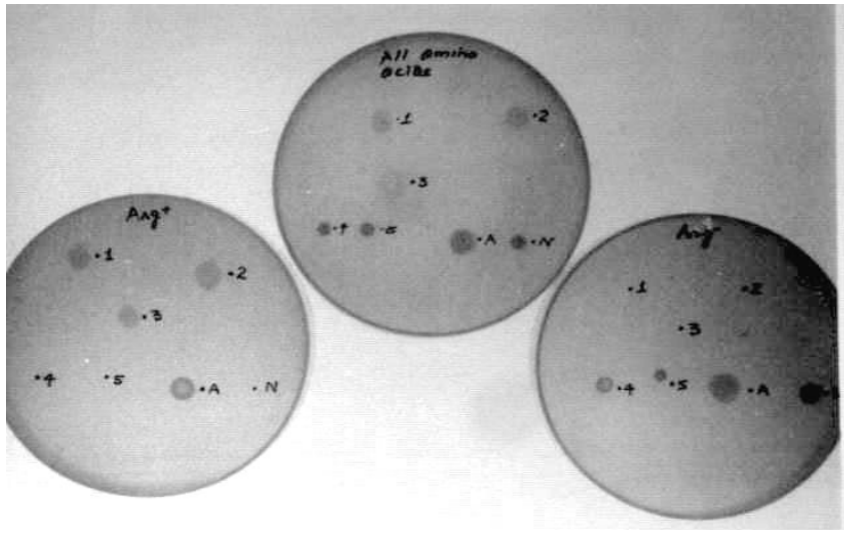

Figure 2

Detection of Auxotrophy of $P$. aeruginosa. Isolate No: Patient PCC.No. I: PCC.No. 499/2000; 2-5: PCC.No. I80/95, A: $P$. aeruginosa ATCC 27853; N: $P$. aeruginosa NCTC 50184(meth-). Plate A: Growth of $\boldsymbol{P}$. aeruginosa on MAM supplemented with arginine only, Plate B: Growth of $P$. aeruginosa on MAM supplemented with all 22 amino acids Plate $\mathbf{C}$ : Growth of $\boldsymbol{P}$. aeruginosa on MAM supplemented with all 22 amino acids except arginine,

to prototrophs $(\mathrm{p}<0.05)$. Three auxotrsophs (isolate No. 1-3) were multidrug resistant (A6 antibiogram) and the producers of ESBL. The reproducible results were obtained on repeating the experiment thrice.

\section{Molecular characterization of P. aeruginosa isolates} Molecular characterization of $P$. aeruginos $a$ isolates $(\mathrm{N}=$ 350) recovered from 27 patients infected with $P$. aeruginos $a$ was achieved by Enterobacterial Repetitive Intergenic Consensus Polymerase Chain Reaction (ERIC-PCR) and PCR-ribotyping. 
Table 3: Phenotypic and genotypic characteristics of $P$. aeruginosa isolated from cystic fibrosis patients (Total No. of patients infected with $P$. aeruginosa $=27$ )

\begin{tabular}{|c|c|c|c|c|c|c|c|c|}
\hline \multirow[t]{2}{*}{ S.No. } & \multirow[t]{2}{*}{ PCC.No. } & \multirow[t]{2}{*}{ Total isolates } & \multicolumn{4}{|c|}{ Phenotypic features } & \multicolumn{2}{|c|}{ Genotypic pattern } \\
\hline & & & Colony morphotype & Antibiogram & ESBL & Auxotrophy & ERIC-PCR & PCR-ribotyping \\
\hline \multicolumn{9}{|c|}{ Group I } \\
\hline I & $180 / 95$ & 146 & Type I-6 & $\mathrm{Al}-\mathrm{A} 4, \mathrm{~A} 6-\mathrm{A} 8$ & + & + & EI-E4 & PI-P4 \\
\hline 2 & $179 / 93$ & 59 & Type 1,2,5 & Al-A5, A7 & - & - & E5-E7 & PI, P5 \\
\hline 3 & $174 / 97$ & 22 & Type I & $\mathrm{Al}$ & - & - & E8 & PI \\
\hline 4 & $308 / 98$ & 22 & Type I-3, 5 & $\mathrm{~A} 1, \mathrm{~A} 2, \mathrm{~A} 5$ & - & - & E9 & $\mathrm{PI}$ \\
\hline 5 & $10 / 99$ & 22 & Typel, 3 & $\mathrm{Al}, \mathrm{A} 2, \mathrm{~A} 4, \mathrm{~A} 5$ & - & - & EIO, EII & PI \\
\hline 6 & $160 / 98$ & 19 & Typel, 3,5 & $\mathrm{~A} 3-\mathrm{A} 5, \mathrm{~A} 7, \mathrm{~A} 8$ & + & - & $\mathrm{E} I 2, \mathrm{EI} 3$ & PI, P2 \\
\hline 7 & $121 / 99$ & 19 & Type I-3, 5 & $\mathrm{~A} 1, \mathrm{~A} 2$ & - & - & EI4 & $\mathrm{PI}$ \\
\hline 8 & $308 / 00$ & 4 & Type $2,3,5$ & $\mathrm{AI}, \mathrm{A} 4$ & - & - & EI5 & $\mathrm{PI}$ \\
\hline \multicolumn{9}{|c|}{ Group2 } \\
\hline 9 & $167 / 98$ & 4 & Typel & AI & - & - & El6 & $\mathrm{PI}$ \\
\hline 10 & $250 / 99$ & 4 & Typel & $\mathrm{Al}$ & - & - & EI7 & $\mathrm{PI}$ \\
\hline 11 & $5 / 00$ & 4 & Typel & Al & - & - & E18 & $\mathrm{PI}$ \\
\hline 12 & $256 / 99$ & 3 & Typel & $\mathrm{A} 1, \mathrm{~A} 2$ & - & - & EI9 & $\mathrm{PI}$ \\
\hline 13 & $337 / 98$ & 3 & Typel & Al & - & - & E20 & $\mathrm{PI}$ \\
\hline 14 & $38 / 95$ & 2 & Typel & Al & - & - & E2I & $\mathrm{PI}$ \\
\hline 15 & $202 / 98$ & 2 & Typel & $\mathrm{Al}, \mathrm{A} 3$ & - & - & E22 & $\mathrm{PI}$ \\
\hline 16 & $312 / 99$ & 2 & Typel & Al & - & - & E23 & $\mathrm{PI}$ \\
\hline 17 & $170 / 99$ & 2 & Typel & $\mathrm{Al}$ & - & - & E24 & $\mathrm{PI}$ \\
\hline 18 & $499 / 00$ & 2 & Typel & A6 & + & + & $\mathrm{EI}$ & $\mathrm{PI}$ \\
\hline 19 & $302 / 96$ & I & Typel & $\mathrm{Al}$ & - & - & E25 & $\mathrm{PI}$ \\
\hline 20 & $95 / 96$ & I & Typel & $\mathrm{Al}$ & - & - & E26 & $\mathrm{PI}$ \\
\hline 21 & $82 / 96$ & 1 & Typel & Al & - & - & E27 & $\mathrm{PI}$ \\
\hline 22 & $8 / 97$ & I & Typel & $\mathrm{Al}$ & - & - & E28 & $\mathrm{PI}$ \\
\hline 23 & $203 / 99$ & I & Typel & Al & - & - & E29 & $\mathrm{PI}$ \\
\hline 24 & $133 / 99$ & 1 & Typel & $\mathrm{Al}$ & - & - & $\mathrm{E} 30$ & $\mathrm{PI}$ \\
\hline 25 & $124 / 00$ & 1 & Typel & $\mathrm{Al}$ & - & - & E3I & $\mathrm{PI}$ \\
\hline 26 & $452 / 00$ & 1 & Typel & Al & - & - & E32 & $\mathrm{PI}$ \\
\hline 27 & $76 / 01$ & 1 & Typel & $\mathrm{Al}$ & - & - & E33 & $\mathrm{PI}$ \\
\hline
\end{tabular}

Genta: Gentamicin; Cipro: Ciprofloxacin; Pipra: Piperacillin; CAZ: Ceftazidime CAZ: S: Sensitive; R: Resistant +: Indicates "and"

\section{ERIC-PCR}

A total of 33 ERIC-PCR (E1-E33) patterns were constructed (Table 3). All 27 patients infected with P. aeruginosa harbored different genotypes with their unique ERICPCR pattern except one patient (PCC.No. 499/00) who was colonized with the similar genotype (E1). Out of 8 patients who were chronically infected with $P$. aeruginosa, 4 patients (PCC.No.180/95, 179/93, 160/98 and 10/99) were colonized with 2-4 genotypes (Fig. $3 \mathrm{~A} \& 3 \mathrm{~B}$ ). The remaining 23 patients (Group1 $=4$ and Group $2=19$ patients) were colonized with a single genotype.

\section{PCR-ribotyping}

A total of 5 PCR ribotyping patterns (P1-P5) were obtained (Fig. 4A \&4B). The molecular weight (bp) of each PCR-ribotyping pattern was P1:570, P2: 730, P3: 1500 \&610, P4: 600 and P5: 570\&130. Twenty four of 27 patients harbored $P$. aeruginos $a$ with a single PCR-ribotyping Pattern P1 and 3 patients (PCC.No. 180/95, 160/98 and 179/93) were colonized with P. aeruginosa with 2 or more PCR-ribotyping patterns. All 3 patients were chronically infected with the organism. Isolates from patient PCC.No 180/95 Pattern P1-P4, PCC.No. 160/98 Pattern P1 and P2 and patient PCC.No. 179/93 Pattern P1 and P5.

By ERIC-PCR, P. aeruginosa isolates from 26/27 patients revealed different genotypes (E1-E33) while by PCRribotyping, 24/27 patients revealed a single PCR-ribotyping pattern (Pattern P1).

Table 3 shows the compilation of results on the patient basis including the phenotypic and genotypic characteristics of each patient.

\section{Discussion}

Cystic fibrosis (CF) is the most common inherited fatal disease in Caucasian population. This generalized disor- 


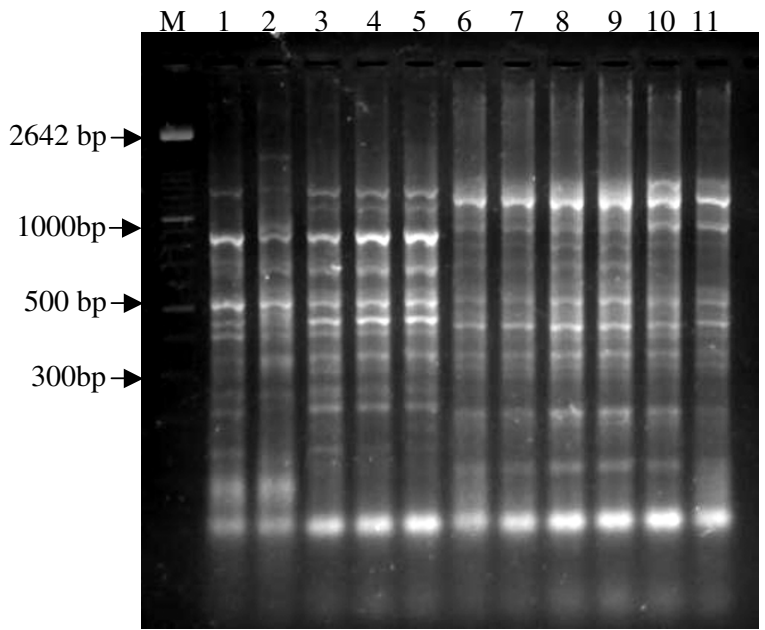

A

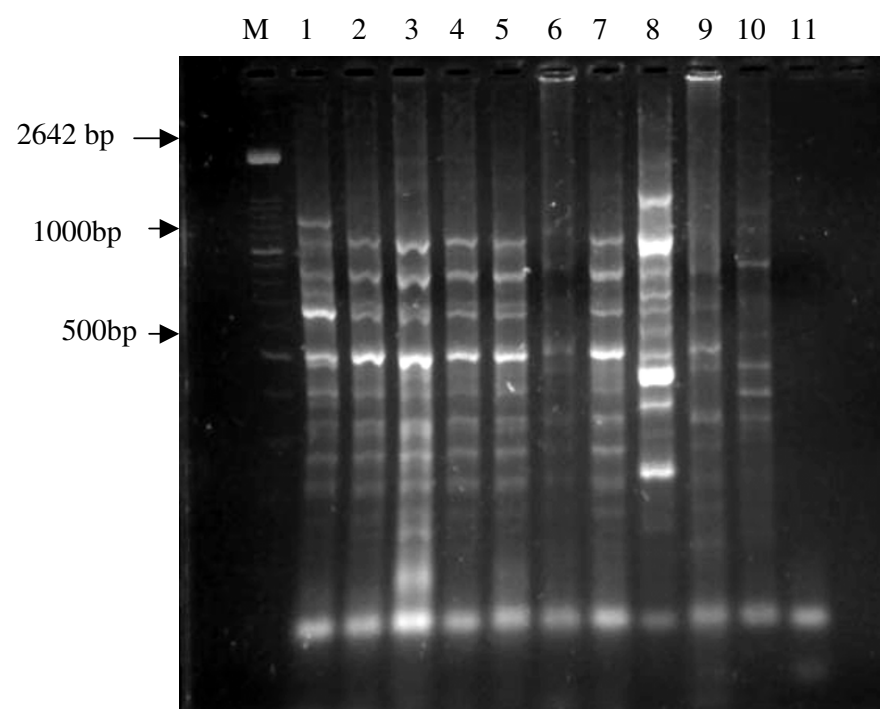

B

\section{Figure 3}

ERIC-PCR patterns of $P$. aeruginosa isolates from the patient A. PCC. No. I 80/95. $\bullet$ Lane M : I00 bp ladder, • Lane I : Pattern EI, • Lane 2 : Pattern E3, • Lane 3-5 : Pattern E I, • Lane 6-I I : Pattern E2. B. PCC.No. I 80/95 and I 79/93 - Lane M : 100 bp ladder, • Lane I-7 (PCC.No. I80/95) : Pattern E4, • Lane 8 (PCC. No. I 79/93) : Pattern E5, - Lane 9 (PCC.No.I 80/95) : Pattern E4, - Lane 10 : P. aeruginosa ATCC $27853 \cdot$ Lane I I : Negative control

der of salt and water transport of exocrine glands is caused by mutations in cystic fibrosis transmembrane regulator gene in the airway epithelial cells and submucosal glands. These patients are very prone to bronchopulmonary infection due to lack of the innate defensive mechanisms. Pulmonary infection is the most common cause of morbidity and mortality in CF patients [16].

CF was thought to be extremely rare in India. However, recent reports from Indian subcontinent suggested CF to be more common in Indian population than what was previously thought [1]. In our study, we have followed all 56 patients attending the Pediatric Chest Clinic at All India Institute Of Medical Sciences, New Delhi, INDIA between August 1998-August 2001. The median age of diagnosis of patients was 4.5 years, which was much higher than what was earlier reported in the literature where the median age was found as 6 and 12 months among Caucasian American and Indian American children respectively [17]. This may be due to delayed diagnosis of CF patients in India.

P. aeruginosa has been the predominant bacterium associated with pulmonary infection in patients with CF [1822]. In our study, P. aeruginosa was also found as an implicated organism in $82 \%$ culture positive patients and its rate of infection was significantly higher as compared to the other organisms in CF patients. The colonization with $P$. aeruginosa has been linked with pulmonary deterioration of CF patient resulting in an increased risk of hospitalization [21]. In the study, all the patients $(n=9)$ who required hospitalization were chronically infected with $P$. aeruginosa.

Besides morbidity, $P$. aeruginosa has been associated with increased mortality [18-22]. In the study all the 4 patients who died were chronically infected with $P$. aeruginosa. Thus significant higher rates of hospitalization and death were observed among patients chronically infected with $P$. aeruginosa.

Although prolonged and frequent administration of antibiotics has been useful in the treatment of pulmonary exacerbations, there is risk of development of multiple drug resistance in $P$. aeruginosa strains. In the study, $41 \%$ isolates were multidrug resistant and were recovered from chronically infected patients, indicating the possible role of antibiotic pressure. Similar findings have been reported elsewhere [23-25].

All three patients infected with ESBL positive isolates were found sensitive to meropenam and imipenam. The simi- 


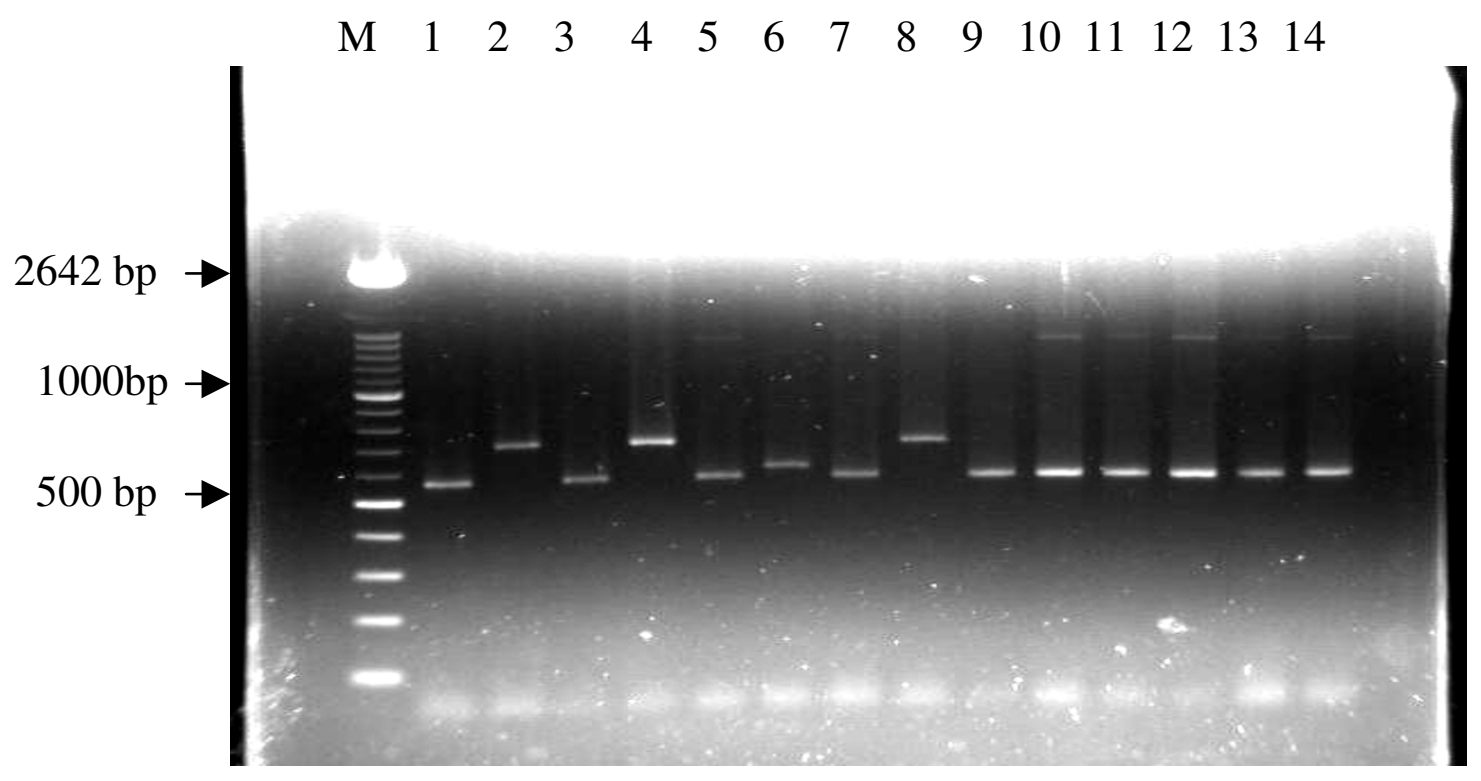

A

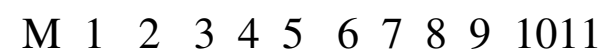

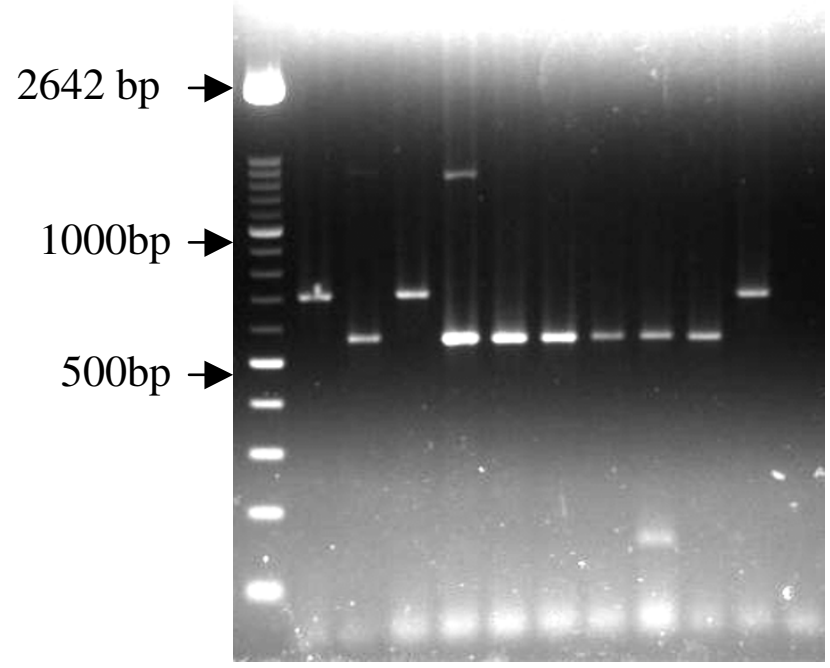

B

\section{Figure 4}

PCR-ribotyping of $\boldsymbol{P}$. aeruginosa isolates from the patient A. PCC.No. 180/95 • Lane M : 100 bp ladder, $\bullet$ Lane I, 3,7 and 9 : Pattern PI, • Lane 2, 4 and 8 : Pattern P2, • Lane 5, I0-I4 : Pattern P3, • Lane 6 : Pattern P4B. PCC.No. I80/95 and I79/93 - Lane M : 100 bp ladder, $\bullet$ Lane I,3 and IO (PCC.No.I80/95) : Pattern P2, • Lane 2,4 (PCC.No.I80/95) : Pattern P4, • Lane 5-7 and 9 (PCC.No.I 80/95) : Pattern PI, • Lane 8 (PCC.No. I 79/93 : Pattern P5, • Lane I I : Negative control 
lar finding has been reported earlier where carbapenams have been found the most effective and reliable against ESBL positive isolates [26].

During chronic infection with the organism, there is progressive anatomical deterioration of the CF lung, which provides a highly spatial structured environment leading to diversification of the organism into six different colony morphological types-Type1-Type 6[12]. This diversification enhances the capacity of an organism to survive in lower respiratory tract of CF patients [13]. In the present study, $7 / 8(87.5 \%)$ chronically infected patients harbored different colony morphological types in the sputum samples.

Once $P$. aeruginos $a$ has colonized the respiratory passages of CF patients, it leads to the induction of mucoidity, resulting in persistent infections. In the study, 6/8(75\%) patients who were chronically infected with $P$. aeruginosa harbored mucoid $P$. aeruginosa in their respiratory samples. Our finding in agreement with the earlier reports where an increased association of mucoidity with chronicity of infection was observed $[4,27,28]$. Out of 4 patients who died, 3(75\%) harbored mucoid colonies in their respiratory samples signifying the association of mucoidy with high mortality in CF patients. Our finding was in accordance with the earlier study where association of mucoidity with high risk of death was observed [26].

The antimicrobial susceptibility among mucoid and nonmucoid isolates was compared. The mucoid isolates showed increased resistance against gentamicin and lower resistance against beta lactam (7.4\% to piperacillin and no resistance to ceftazidime). The results were analogy to the studies where high resistance to aminoglycosides $[29,30]$ and low resistance to beta lactam $[31,32]$ was observed among mucoid isolates.

With chronicity of infection, there is an increase in concentration of amino acids in bronchial secretions, which is due to active epithelial transport, plasma exudation and protease activity in lung tissue and surface fluid. This results in development of auxotrophy for certain amino acids required for growth. In the present study, auxotrophic $P$. aeruginosa was found in $2 / 27(7.4 \%)$ patients in contrary to an earlier report where auxotrophy of $P$. aeruginosa was found in $86 \%$ patients infected with the organism [33]. The reason for low percentage of auxotrophs among our patients may be due to lower number of chronic patients in this study.

Furthermore in the study, 3/5 auxotrophs had arginine requirement and the remaining 2 were auxotrophic for methionine. This was in agreement with the earlier reports where methionine and arginine were considered as the most commonly required amino acids by auxotrophs $[14,33,34]$. As these strains could be more resistant to antibiotics, it is important to study auxotrophy of isolates in CF patients with severe $P$. aeruginosa infection in order to plan out appropriate therapy for the management of $\mathrm{CF}$ patients.

Bacterial typing schemes are based on phenotypic or genotypic analysis of multiple isolates within a particular species. Such analysis helps in investigation of outbreaks and determines the recurrence or relapse of an infection. The epidemiology of $P$. aeruginos a infection in CF patients has been hampered since the conventional phenotyping methods-phage and pyocin typing have poor discriminatory power and poor reproducibility respectively. Genetic typing methods have shown to be more discriminatory than phenotypic methods for typing $P$. aeruginosa CF isolates [35]. Various genotypic typing methods- restriction fragment length polymorphism (RFLP) analysis of tox A I and pil A loci, pulse field gel electrophoresis (PFGE), random amplified DNA polymorphism (RAPD) PCR have been used to identify the number of strains colonizing the CF patient [13].

PCR-ribotyping is based on the uniform spacer region in rRNA operons of $P$. aeruginosa [36] resulting in low degree of heterogeneity of rRNA operons. In the study, majority of isolates showed the single PCR-ribotyping Pattern P1, probably due to the above reason. Hence for the study of dynamic of $P$. aeruginosa strain variation, ERIC-PCR may be a useful tool.

By ERIC-PCR, all 27 culture positive patients except one were found colonized with the unique genotype (E1E33). This indicates that cross-colonization among unrelated CF patients was uncommon. The results were concordant to the earlier studies where cross-infection between unrelated patients was considered unusual $[37,38]$. All 27 patients except 4 were colonized with the single persistent genotype. These 4 patients were chronically infected and were colonized with 2 or more genotypes. Our results were in agreement with the previous studies where majority of CF patients were colonized with a predominant genotype for longer period of time but high degree of genotypic variability within patients have been observed suggesting that the colonizing strain may occasionally be replaced [13,39-41].

Thus we have found that CF is a problem in our country but delayed diagnosis and low index of suspicion have delayed the appropriate management of these patients. Although all the patients attending the Pediatric chest clinic were included in the study, the number of patients, especially the chronic ones, was limited in India, which further limited the number of chronic patients. Although 
the small number of patients in our study is not sufficient to answer all the epidemiological questions, these results indicate that a larger scale CF study should be conducted in India.

\section{Conclusion}

$P$. aeruginosa is the major colonizer of the respiratory passages of CF patients. The patients with chronic infection showed significantly higher rates of hospitalization and death. With chronicity of infection, the organism undergoes phenotypic and genotypic variations. Among phenotypic variations, the organism becomes multidrug resistant, diversifies its colony morphology, acquires mucoidity and becomes auxotrophic for various amino acids. The multiple genotypes have been found to colonize the respiratory passages of CF patients.

Thus in a particular clinical set up, high index of suspicion should be there for diagnosis of CF patients so as to prevent the delay in diagnosis and management of $\mathrm{CF}$ patients. The routine microbiological examination of respiratory samples from CF patients are advised to study the characteristics of the infecting agents of which $P$. aeruginosa is the most common.

\section{Methods \\ Subjects}

All patients with CF (age equal to or less than 15 years) attending the Pediatric Chest Clinic at All India Institute Of Medical Sciences between August 1998-August 2001 were included in the study. There was a regular monthly follow-up of each patient to the clinic. Patients who required hospitalization were admitted to the Pediatric ward and were followed daily. The diagnosis of CF was based on both clinical and laboratory parameters [42]. For analysis, patients were divided into 2 groups -Group 1 and Group 2 based on the chronicity of infection with $P$. aeruginosa [12]. Patients under Group 1 were chronically infected with $P$. aeruginosa for the past two years and Group 2 included patients who were intermittently colonized with the organism.

\section{Sample collection}

During each visit of a patient to the clinic and during the period of hospitalization, a representative sample - sputum and/or throat swab after physiotherapy was collected [41]. The sputum sample was collected directly in a sterile vial after coughing. Throat swab was considered as an alternative respiratory sample and was collected from children who were either below 2 years of age or were not able to expectorate sputum [43].

\section{Isolation and characterization of $P$. aeruginosa strains}

The sputum was vortexed (for complete homogenization) and cultured on Chocolate agar, Blood agar containing
5\% sheep blood and Mac Conkey agar (Hi Media, Mumbai). The plates were incubated at $37^{\circ} \mathrm{C}$ for 18 hrs. $P$. aeruginosa was identified by standard bacteriological methods [44] and its different colony morphotypes were recorded [12].

\section{Antibiotic susceptibility Antibiogram}

The single representative from each colony morphotype was taken and tested for antimicrobial susceptibility by agar dilution method as per NCCLS guidelines [45]. Of the six colony morphotypes, Type 5 was the mucoid morphotype and the rest morphotypes(Type 1-4 and Type 6) were the nonmucoid morphotypes. Depending on antimicrobial susceptibility of an organism, antibiograms were constructed where intermediate and resistant categories were clubbed. The antibiotics tested were gentamicin, ciprofloxacin, piperacillin and ceftazidime. All these antibiotics were procured from Hi Media, Mumbai. $P$. aeruginosa ATCC 27853 and Escherchia coli ATCC 25922(ATCC, USA) served as the control strains. Those isolates, which showed resistance to two or more than two antibiotics were considered multidrug resistant [10].

Production of Extended spectrum beta lactamase (ESBL) Those isolates, which showed resistance to ceftazidime (CAZ) were tested for ESBL production by Disc Diffusion according to NCCLS guidelines [46] and by Etest method (AB BIODISK, Sweden). All ESBL positive isolates were further tested for carbapenam-imipenam and carbapenam susceptibilty by disk diffusion method [46].

\section{Phenotypic characterization of $\mathbf{P}$. aeruginosa isolates}

Different phenotypic features of $P$. aeruginos a were studied in CF patients. The phenotypic features included colony morphotypes and auxotrophy.

\section{Colony morphotypes}

Based on colony morphology of the organism, there are six different colony morphotypes of $P$. aeruginosa [12]. The presence of six different morphotypes was observed in respiratory samples of CF patients. Type 5 morphotype showed mucoidy, which is one of the virulence factor of the organism.

\section{Auxotrophy}

All $P$. aeruginosa isolates from CF patients were tested for auxotrophy. The inoculum was adjusted to $0.5 \mathrm{Mc}$ Farland and diluted to have the final concentration of $10^{4} \mathrm{cells} / \mu \mathrm{l}$. One microlitre of the inoculum was spotted on Mueller Hinton agar (MHA) and Minimal agar medium (MAM) where MHA served as the complex medium and MAM as nutrient deficient medium. The plates were incubated at $37^{\circ} \mathrm{C}$ for 48 hrs. Those isolates, which did not grow on MAM plate but grew on MHA were considered auxo- 
trophs. Those isolates, which were able to grow on both MHA and MAM plates were considered prototrophs. $P$. aeruginosa NCTC 50184 (meth -) and P. aeruginosa ATCC 27853 were included as the known auxotrophic and prototrophic strain respectively. The specific amino acid requirements were identified.

\section{Identification of specific amino acid requirements}

The stock solution of all 23 L-amino acids including cysteine, proline, lysine, arginine (Sigma, USA), cystine, methionine, serine $(\mathrm{CDH})$, glutamine, glutamic acid, glycine, histidine, hydroxy-proline, isoleucine, leucine, ornithine, phenylalanine, alanine, asparagine, aspartic acid, threonine, tryptophan, tyrosine and valine (HiMedia, Mumbai) were prepared at the concentration of $2 \mathrm{mg} / \mathrm{ml}$ in either sterile distilled water or the appropriate solvent. All the amino acids were filter sterilized by $0.45 \mu \mathrm{m}$ Millipore filter (Millipore, USA). The specific amino acid/ acids requirement was determined by two methods [34].

The first method helps in detection of single or multiple amino acids. In this method, a single amino acid at the concentration of $20 \mu \mathrm{g} / \mathrm{ml}$ was added to each MAM plate. The plates were inoculated and incubated at $37^{\circ} \mathrm{C}$ for 48 hrs. The growth on specific amino acid containing plates indicated the requirement for single/multiple amino acids while no growth in any of the MAM plate supplemented with amino acid indicated either the requirement for two or more amino acids in combination or some other growth factor.

The second method helps in detection of combination of amino acids. All amino acids (at the concentration of 20 $\mu \mathrm{g} / \mathrm{ml}$ ) less one were added to molten MAM. The plates were inoculated and incubated as described above. The specific requirement for an amino acid/combination of amino acids was evident, if the isolate failed to grow on agar not containing that particular amino acid.

\section{Antibiotic resistance}

The antibiotic susceptibility of auxotrophs and prototrophs was determined by agar dilution method and the antibiotic resistance was compared between them. The antibiotics tested were gentamicin, ciprofloxacin, piperacillin and ceftazidime.

\section{Molecular characterization of $P$. aeruginosa isolates}

Molecular typing of $P$. aeruginosa isolates from all culture positive patients was done by ERIC-PCR and PCR-ribotyping. Both the molecular methods were carried out as described earlier [12].

\section{Statistical analysis}

To see the association between the categorical variables, Chi square test (with Yates correction)/Fischer's exact test was used. The result was considered significant at $5 \%$ level of significance $(\mathrm{p}<0.05)$. SAS 8.0 statistical package was used for analysis.

\section{Authors' contributions}

GA: Technical procedures, study design and manuscript preparation.

AK: Infrastructure, time-to-time guidance and participated in microbiological experiments.

BKD: Involved in the molecular studies.

SKK: Helped in collection of the samples. Provided case histories of CF patients and helped in evaluation of clinical data.

SND: Performed the statistical analysis.

\section{Acknowledgements}

This work was supported by the Indian Council of Medical Research, New Delhi, INDIA

\section{References}

I. Ahuja AS, Kabra SK: Cystic fibrosis: Indian experience. Ind Pediatr 2002, 39:813-818.

2. Singh $M$, Prasad $R$, Kumar L: Cystic fibrosis in north Indian children. Ind J Pediatr 2002, 69:627-629.

3. Kabra SK, Kabra M, Lodha R, Shastri S, Ghosh M, pandey RM, Kapil A, Aggarwal G, Kapoor V: Clinical profile and frequency of delta $\mathbf{5 0 8}$ mutation in Indian children with cystic fibrosis. Ind Pediatr 2003, 40:612-619.

4. Koch C, Hoiby N: Pathogenesis of cystic fibrosis. Lancet 1993, 341:1065-1069.

5. Kulczycki LL, Murphy TM, Bellanti JA: Pseudomonas colonization in cystic fibrosis. JAMA 1978, 240:30-34.

6. Friend PA: Pulmonary infection in cystic fibrosis. $J$ Infect 1986, I3:55-72.

7. Govan JR, Deretic V: Microbial pathogenesis in cystic fibrosis:mucoid Pseudomonas aeruginosa and Burkholderia cepacia. Microbiol Rev 1996, 60:539-74.

8. Mearns MB, Hunt GH, Rushworth R: Bacterial flora of respiratory tract in patient with cystic fibrosis. Arch Dis Child 197I, 47:902-07.

9. Cheng K, Smith R, Govan JRW, Catherine D, Winstanley Denning N: Spread of beta lactam resistant Pseudomonas aeruginosa in a cystic fibrosis clinic. Lancet 1996, 348:639-42.

10. Saiman L, Mehar F, Niu W, Neu HC, Shaw KJ, Millar G, Prince A: Antibiotic susceptibility of multiply resistant Pseudomonas aeruginosa isolated from CF patients, including transplant candidates. Clin Infect Dis 1996, 23:532-537.

II. Oliver A, Canton R, Baquero F, Blazquez J: High frequency of Hypermutable $P$. aeruginosa in cystic fibrosis lung infection. Science 2000, 288: I25I-I253.

12. Agarwal G, Kapil A, Kabra SK, Chandra R, Das B; Dwivedi SN: Phenotypic and Genotypic variants of Pseudomonas aeruginosa isolated from Cystic Fibrosis Children in India. Ind J Med Res 2002, I | 6:73-8I.

13. Mahenthiralingam E, Campbell ME, Foster J, Lam JS, Speert DP: Random Amplified DNA Typing of Pseudomonas aeruginosa Isolates Recovered from Patients with Cystic Fibrosis. J Clin Microbiol 1996, 34: I I29-I I35.

14. Barth AL, Pitt TL: The high amino acid content of sputum from cystic fibrosis patients promotes the growth of auxotrophic P. aeruginosa. J Med Microbiol 1996, 45: I I0-II9.

15. Belkum AV, Renders NHM, Smith S, Overbeek SE, Verbrugh HA: Comparison of conventional and molecular methods for the detection of bacterial pathogens in sputum samples from 
cystic fibrosis patients. FEMS Immuno Med Microbiol 2000, 27:5I-57.

16. Cystic Fibrosis Foundation: Patient Registry 1998 annual data repot. Bethesda MD: Cystic fibrosis Foundation; 1999.

17. Power CA, Potter EM, Wessel HU, Lloyd Still JD: Cystic fibrosis in Asian Indians. Arch Pediatr Adolesc Med 1996, 150:554-555.

18. FitzSimmons S: The changing epidemiology of cystic fibrosis. Pediatr 1993, I 22: I-9.

19. Govan JRW, Nelson JW: Microbiology of lung infections in cystic fibrosis. Br Med Bull 1992, 48:912-930.

20. Gilligan PH: Microbiology of airway disease in patients with cystic fibrosis. Clin Microbiol Rev I991, 4:35-51.

21. Fick RB Jr: Pathogenesis of Pseudomonas aeruginosa lung lesion in cystic fibrosis. Chest 1989, 96:158-164.

22. Barth AL, Pitt TL: Microbial pathogens associated with Cystic fibrosis: special focus on P. aeruginosa. Braz J Infect Dis 1998, 2:43-6I.

23. Burns JL, Ramsey BW, Smith AL: Clinical manifestations and treatment of pulmonary infections in cystic fibrosis. Adv Pediatr Infect Dis 1993, 8:53-66.

24. Mouton JW, Hollander JGD, Horrevorts AM: Emergence of antibiotic resistance amongst $P$. aeruginosa isolates from patients with cystic fibrosis. I Antimicrob Chemother 1993, 31:919-926.

25. Ogle JW, Reller LB, Vasil ML: Development of resistance in $\boldsymbol{P}$. aeruginosa to imipenam, norfloxacin and ciprofloxacin during therapy: proof provided by typing with a DNA probe. J Infect Dis 1988, 157:743-8.

26. Kfoury JNS, Araj GF: Recent developments in $\beta$ lactamases and extended spectrum $\beta$ lactamases. BMJ 2003, 327: I 209-13.

27. Pederson SS: Lung infection with alginate producing, mucoid Pseudomonas aeruginosa in cystic fibrosis. APMIS 1992, 28: I-79.

28. Thomassen MJ, Demko CA, Boxerbaum B, Stern RC, Kuchenbrod PJ: Multiple isolates of Pseudomonas aeruginosa with differing antimicrobial susceptibility patterns from patients with cystic fibrosis. J Infect Dis 1979, 140:873-80.

29. Govan JRW, Fyfe JAM: Mucoid Pseudomonas aeruginosa and cystic fibrosis: resitance of the mucoid form to carbenicillin, flucloxacillin, and tobramycin and the isolation of mucoid variants in vitro. J Antimicrob Chemother 1978, 4:233-240.

30. Demko CA, Thomassen : Effect of mucoid property on antibiotic susceptibility of $P$. aeruginosa. Curr Microbiol I980, 4:69-73.

31. Tannenbaum CS, Hastie AT, Higgins ML, Kueppers F, Weinbaum G Inability of purified Pseudomonas aeruginosa exopolysaccharide to bind selected antibiotics. Antimicrob Agents Chemothe 1984, 25:673-5.

32. Nicholas WW, Evans MJ, Slack MPE, Walmsley HL: The penetration of antibiotics into aggregrates of mucoid and nonmucoid $P$. aeruginosa. J gen Microbiol 1989, 135:129|-1303.

33. Taylor RFH, Hodson ME, Pitt TL: Adult cystic fibrosis association of acute pulmonary exacerbation and increasing severity of lung disease with auxotrophic mutants of $P$. aeruginosa. Thorax 1993, 48: 1002-5.

34. Barth AL, Pitt TL: Auxotrophic variants of Pseudomonas aeruginosa are selected from prototrophic wild type strains in respiratory infections in patients with cystic fibrosis. J Clin Microbiol 1995, 33:37-40.

35. The International P. aeruginosa Typing Study Group: A multicenter comparison of methods for typing strains of $P$. aeruginosa predominantly from patients with cystic fibrosis. J Infect Dis 1994, 169:134-142.

36. Severino $P$, Darini AL, Magalhaes VD: The discriminatory power of ribo-PCR compared to conventional ribotyping for epidemiological purposes. APMIS 1999, 107:1079-84.

37. Speert DP, Campbell ME: Hospital epidemiology of Pseudomonass aeruginosa from patients with cystic fibrosis. J Hosp Infect 1987, 9:1|-21.

38. Speert DP, Lawton D, Damm S: Communicability of Pseudomonas aeruginosa in a cystic fibrosis summer camp. J Pediatr 1982, I 01:227-229.

39. Renders NHM, Sijmons MAF, Van Belkum A, Overbeek E, Mouton JW, Verbrugh HA: Exchange of Pseudomonas aeruginosa strains among cystic fibrosis siblings. Res Microbiol 1997, I 48:447-454.

40. Silbert S, Barth AL, Sadar HS: Heterogeneity of $\boldsymbol{P}$. aeruginosa in Brazilian Cystic Fibrosis Patients. J Clin Microbiol 200I, 39:3976-398I.
4I. Burns JL, Gibson RL, McNamara S, Yuin D, Emerson J, Rosenfeld M, et al.: Longitudinal assessment of Pseudomonas aeruginosa in young children with cystic fibrosis. J Infect Dis 200I, 183:444-52.

42. Boat TF, Wesh MJ, Beaudet A, et al.: Cystic Fibrosis. In The metabolic basis of inherited diseases Edited by: Scriver CR, Beaudet AL, Sly WS, Valle D, Stansbury JB, Wyngaorden JB. New York: Mc GrawHill; 1989:2649-2680

43. Kabra SK, Alok A, Kapil A, Aggarwal G, Kabra M, Lodha R, et al.: Can Throat Swab after physiotherapy replace sputum for identification of microbial pathogens in children with cystic fibrosis? Ind J Ped 2004, 7 I:2 I-23

44. Govan JRW: Pseudomonas, Stenotrophomonas, Burkholderia. In Practical Medical Microbiology 14th edition. Collee JG, Fraser AG, Marmion BP and Simmons A. New York: Churchill Livingstone; 1996:413-424.

45. National Committee for Clinical Laboratory Standards: MIC testing-supplement tables. Approved standard MI00-SI0(M7). National Committee for Clinical Laboratory Standards, Wayne, Pa; 2000.

46. National Committee for Clinical Laboratory Standards: Performance standards for antimicrobial disk susceptibility tests. Approved standard M2-A7. NCCLS Document M2-A7. 7th edition. National Committee for Clinical Laboratory Standards, Wayne, Pa; 2000.
Publish with Biomed Central and every scientist can read your work free of charge

"BioMed Central will be the most significant development for disseminating the results of biomedical research in our lifetime. "

Sir Paul Nurse, Cancer Research UK

Your research papers will be:

- available free of charge to the entire biomedical community

- peer reviewed and published immediately upon acceptance

- cited in PubMed and archived on PubMed Central

- yours - you keep the copyright
BioMedcentral 